Fachbereich Mathematik

Nr. $160 / 93$

\title{
A Geometric Cauchy Problem for Timelike Minimal Surfaces
}

\author{
Thomas Deck \\ Lehrstuhl für Mathematik I \\ Universität Mannheim \\ D - 68131 Mannheim, A5 \\ Germany
}

\begin{abstract}
We investigate two-dimensional timelike surfaces $\Sigma$ in a spacetime $(X, g)$. It is shown that orientable surfaces, with two spacelike boundary components $\gamma$ (homeomorphic to $S^{1}$ ) are necessarily of topological type $[0,1] \times S^{1}$. We treat the initial value problem of a string (known from physics) as a purely geometric problem: Find a minimal surface $\Sigma$ which is specified by an initial curve $\gamma$ and by a distribution of timelike tangent planes along $\gamma$. We prove local existence and uniqueness of $\Sigma$ and also obtain global existence for special types $(X, g)$. Global existence does not generally hold; we give a counter-example which can be interpreted as a string collapsing into a black hole.
\end{abstract}

1991 AMS: 35 L 70, 53 C 42, 83 E 30

September 1993 


\section{Introduction}

Since our considerations are motivated by the theory of classical strings [1], we use that picture as an intuitive guideline. The basics of that theory can be summarized as follows: A closed string is an object $\gamma$ in space that is homeomorphic to $S^{1}, \gamma \cong S^{1}$. During the time evolution a string sweeps out a surface $\Sigma$, called worldsheet, in spacetime $(X, g)$; we generally assume $\operatorname{dim}(X) \geq 3$. For physical reasons $\Sigma$ is supposed to be a timelike surface, i.e. at each $p \in \Sigma$ the tangent plane $T_{p} \Sigma \subset T_{p} X$ is generated by a timelike and by a spacelike vector. The dynamical equations for a string are defined by a variational principle: The first area variation of $\Sigma$ must vanish subject to the condition that the initial and final configuration of the string are held fixed. Hence, $\Sigma$ will be a minimal surface having two spacelike boundary components. The geometric point of view suggests to ask whether such minimal surfaces allow for different topological types; in Euclidean spaces this so-called Douglas problem [2] indeed does. We will show in chapter 1 that for $C^{2}$ immersed surfaces $\Sigma$ only those of topological type $[0,1] \times S^{1}$ can be consistent with the physical requirements on $\Sigma$ to be timelike and to have spacelike boundaries. For later needs we also give a direct construction of conformal parameters on $[0, \infty) \times S^{1}$.

To determine a concrete string motion one has to solve a Cauchy problem whose solution defines the surface $\Sigma$. Generally, the "initial data" for a surface $\Sigma$ are not uniquely fixed, but depend on the specific parametrization of $\Sigma$. To overcome this ambiguity, we formulate the Cauchy problem in purely geometric terms. Let us first assume that we are given a timelike minimal surface $\Sigma \subset X$ which contains a spacelike curve $\gamma \cong S^{1}$ ( $\gamma$ represents the configuration of the string). The tangent planes of $\Sigma$ with base points on $\gamma$ fix the "direction" in $X$, into which the string moves in the following sense: The planes provide a linear approximation of $\Sigma$ in a neighbourhood of $\gamma$, and they represent the velocity distribution along the string. These observations suggest to define a geometric Cauchy problem: Find, for $\gamma$ prescribed, a timelike minimal surface $\Sigma$ containing $\gamma$ such that the tangent planes of $\Sigma$ along $\gamma$ coincide with a given distribution of tangent planes on $\gamma$.

In chapter 2 we simplify the equations of motion by conformal parameters and show how these parameters are used to remove singularities which can arise in these equations. Of course, the geometric singular behaviour cannot be removed; this behaviour is in sharp contrast to the one of minimal surfaces in Riemannian manifolds.

We establish local existence and uniqueness for the geometric Cauchy problem in chapter 3 . We show that global existence of the nonlinear hyperbolic equations of motion holds if $(X, g)$ has a certain product structure. As an illustration we compute in chapter 4 a minimal surface $\Sigma$ in Schwarzschild spacetime*.

* The only other solution in a curved space $I$ found is given in [3]. It describes a closed string interacting with a gravitational wave. 


\section{Topology and parametrization of immersed timelike surfaces}

Let $(X, g)$ be a spacetime, i.e. a time-orientable Lorentz manifold. We consider a twodimensional, compact and orientable manifold $M$ of class $C^{\infty}$, having two disjoint boundary components $\gamma \cong S^{1}$. $M$ is immersed into $X$ by a $C^{k}$-map $i: M \rightarrow X, k \geq 1$, such that the pullback metric $h=i^{*} g$ has signature $(-1,1)$ on $M$, and $i(\partial M)$ consists of two disjoint spacelike curves*. If all these properties are fulfilled we speak, for convenience, of an immersed timelike surface $\left(M, i^{*} g\right)$.

Theorem 1.1: An immersed timelike surface $\left(M, i^{*} g\right)$ is homeomorphic to $[0,1] \times S^{1}$.

Proof: First, we construct nonvanishing vector fields on the double $2 M$ of $M$ : The induced metric $h$ has signature $(-1,1)$ so the set $\mathcal{T}_{p}=\left\{v \in T_{p} M \mid h(v, v)<0\right\}$ consists of two disjoint parts, $\mathcal{T}_{p}=\mathcal{T}_{p}^{+} \cup \mathcal{T}_{p}^{-}$. Let $\mathcal{T}_{p}^{+}$be the future pointing part of $\mathcal{T}_{p}$ with respect to a fixed time orientation of $X$. The forward light cone $\partial \mathcal{T}_{p}^{+}$is generated by two vectors, $\partial \mathcal{T}_{p}^{+}=\left\{\mathbb{R}^{+} \cdot v_{p}\right\} \cup\left\{\mathbb{R}^{+} \cdot w_{p}\right\}$, which form a positive ordered basis $\left\{v_{p}, w_{p}\right\}$ in $T_{p} M(M$ is orientable). We normalize $v_{p}$ and $w_{p}$ with respect to a fixed Riemannian metric on $M$ and obtain an ordered basis $\left\{e_{1}(p), e_{2}(p)\right\}$ of unit vectors for each $p \in M$. The maps $e_{n}: p \rightarrow e_{n}(p)(n=1,2)$ define two nonvanishing $C^{k-1}$-vector fields $e_{1}$ and $e_{2}$ on $M$. Because $\partial M$ is spacelike, the fields $e_{1}$ and $e_{2}$ are transversal to $\partial M$. Thus, nonvanishing continuous vector fields on $2 M$ exist.

This shows that $2 M$ has Euler characteristic zero so it must be a torus $T$. The set $\partial M \subset T$ consists of two disjoint closed curves. Taking into account the fundamental group $\pi(T) \cong \mathscr{Z} \oplus \mathbb{Z}$ one verifies $M \cong[0,1] \times S^{1}$, by simple topological arguments.

Remark: The condition on $i(\partial M)$ to be spacelike is crucial because on noncompact manifolds (especially $M \backslash \partial M$ ) smooth Lorentz metrics always exist. Theorem 1.1 can easily be extended to more complicated situations. For example, there is no timelike surface $M$ having the topology of a sphere with three open discs removed and with spacelike boundary components: The double of $M$ would be a surface of genus two.

The above result suggests that immersed timelike surfaces of type $M_{a}=[0, a] \times S^{1}$ are appropriate for the description of motions of closed strings. The equations of motion simplify considerably in conformal parameters $(\tau, \sigma)$ on $M_{a}$, i.e. $h=i^{*}-g$ has coordinate expression $h(\tau, \sigma)=\Lambda(\tau, \sigma)\left(-d \tau^{2}+d \sigma^{2}\right)$, where $\Lambda$ is a function. Global existence of such parameters is guaranteed by

Proposition 1.2: Let $i: M \rightarrow X$ be a $C^{k}$-map with $M=[0, \infty) \times S^{1}$ and $k \geq 2$. Suppose that $\left(M_{a},\left.h\right|_{M_{a}}\right)$ is an immersed timelike surface, for all $a>0$. Then $(M, h)$ admits a conformal $C^{k-1}$-parametrization with $\tau(\cdot) \in[0, \infty), \sigma(\cdot) \in[0,2 \pi) \bmod 2 \pi$ and $\tau\left(\{0\} \times S^{1}\right)=\{0\}$.

Proof: The $C^{k-1}$-field $e_{1}$ constructed in the proof of Theorem 1.1 has integral curves reaching from points $P_{1} \in\{0\} \times S^{1}$ to $\{a\} \times S^{\mathbf{1}}$, because $e_{1}$ is transversal to each $\{c\} \times S^{\mathbf{1}}$,

* These are physically natural conditions on a worldsheet $\Sigma=i(M)$; $i$ need not be injective because a string is allowed to have self-intersections. 
$c \in[0, a]$. Therefore, the set of integral curves which meet $\{0\} \times S^{1}$ cover $M$ and each $p \in M$ lies on exactly one integral curve of $e_{1}$. The vector field $e_{2}$ has integral curves with similar properties, starting at $P_{2} \in\{0\} \times S^{1}$. This pair of integral curves constitute the coordinate lines of local $C^{k-1}$-coordinate systems around $p \in M$. In such coordinates, say $(u, v)$, the metric $h$ is given by $h(u, v)=f(u, v) d u d v$, because $\partial_{u}$ and $\partial_{v}$ are lightlike vectors. The map $\Psi=\pi_{1} \times \pi_{2}$, assigning to each $p \in M$ the initial points $\left(P_{1}, P_{2}\right) \in S^{1} \times S^{1}$ is locally bijective of class $C^{k-1}$. We parametrize $S^{1}=\left\{e^{i \sigma} \mid \sigma \in \mathbb{R}\right\}$ by $\operatorname{charts} \varphi_{\alpha}\left(e^{i \sigma}\right)=\sigma \in(0,2 \pi)$, $\varphi_{\beta}\left(e^{i \sigma}\right)=\sigma \in(-\pi, \pi)$ and obtain a closed one-form $\omega$ on $S^{1}: \omega=d \varphi_{\alpha}$ resp. $\omega=d \varphi_{\beta}$ on each domain of definition. Then $d \sigma_{1}:=\pi_{1}^{*} \omega$ and $d \sigma_{2}:=\pi_{2}^{*} \omega$ are pointwise linear independent closed one-forms on $M$ of class $C^{k-2}$. The functions $\tilde{\tau}(p):=\frac{1}{2} \int_{p_{0}}^{p}\left(d \sigma_{1}-d \sigma_{2}\right)$ and $\sigma(p):=\frac{1}{2} \int_{p_{0}}^{p}\left(d \sigma_{1}+d \sigma_{2}\right) \bmod 2 \pi\left(p_{0} \in\{0\} \times S^{1}\right.$ fixed) now are conformal parameters with $\tilde{\tau} \geq 0$ or $\tilde{\tau} \leq 0$ on $M$. We set $\tau=\tilde{\tau}$ or $\tau=-\tilde{\tau}$ to achieve $\tau \geq 0$ on $M$.

Remark: Conformal coordinates are not uniquely determined: If we apply a diffeomorphism $\psi: S^{1} \rightarrow S^{1}$ we obtain $\left(\tau^{\prime}, \sigma^{\prime}\right)$ in the same way as above but with $\omega$ replaced by $\psi^{*} \omega$. Intuitively, this means that we can choose the "density" of the integral curves of $e_{1}$ and $e_{2}$ arbitrarily. More generally, the whole construction above holds for $M=\mathbb{R} \times S^{1}$. In that case we have the additional freedom to choose an arbitrary pair of diffeomorphisms $\psi_{\mathrm{I}}$, $\psi_{2}: S^{1} \rightarrow S^{1}$ to define new conformal $\left(\tau^{\prime}, \sigma^{\prime}\right)$-coordinates via $d \sigma_{i}=\pi_{i}^{*} \psi_{i}^{*} \omega$ (then $\tau^{\prime}=0$ does not correspond to $\left.\{0\} \times S^{1}\right)$. The map $\Phi_{\psi_{1} \psi_{2}}:(\tau, \sigma) \rightarrow\left(\tau^{\prime}, \sigma^{\prime}\right)$ obeys $\Phi_{\psi_{1} \psi_{2}}^{*} h=f \cdot h$ ( $f$ a function), i.e. $\Phi_{\psi_{1} \psi_{2}}$ is a conformal coordinate transformation. This provides a clear interpretation for the group action of $\operatorname{Diff}\left(S^{1}\right) \times \operatorname{Diff}\left(S^{1}\right)$ on conformal coordinates.

\section{Timelike minimal surfaces}

First, some notations are given: For $v, w \in T_{p} X$ we set $v w:=g(v, w)=g_{\mu \nu} v^{\mu} w^{\nu}$ (summation convention in coordinates). The flat, two-dimensional Lorentz metric reads $\eta=\operatorname{diag}(-1,1)$. Derivatives with respect to $\left(u^{1}, u^{2}\right)$-coordinates on $M$ are labeled by $\frac{\partial f}{\partial u^{a}}=\partial_{a} f=f_{, a}$. We use local $x^{\mu}$-coordinates on $X$ to represent the map $i: M \rightarrow X$.

The induced metric $h=i^{*} g$ has components $h_{a b}=g_{\mu \nu} x_{, a}^{\mu} x_{, b}^{\nu}$. In conformal parameters on $M$ we obtain $g_{\mu \nu} x_{, a}^{\mu} x_{, b}^{\nu}=\Lambda(\tau, \sigma) \cdot \eta_{a b}$. With $\dot{x}^{\mu}=\frac{\partial x^{\mu}}{\partial \tau}$ and $x^{\mu \prime}=\frac{\partial x^{\mu}}{\partial \sigma_{-}}$these equations are obviously equivalent to

$$
\dot{x}^{2}+x^{\prime 2}=0 \quad, \quad \dot{x} x^{\prime}=0
$$

and for $a=b=0$ we read off $\Lambda=-\dot{x}^{2}$. In the physical literature (2.1) is called the conformal gauge condition (imposed on the surface parametrization).

The area functional of an immersed timelike surface $M$ reads

$$
S=\int_{M} \sqrt{-|h|} d^{2} u \quad, \quad|h|=\operatorname{det}\left(h_{a b}\right) .
$$

Demanding stationarity of $S$, i.e. $\delta S=0$, one obtains a system of coupled Euler-Lagrange equations for the components $x^{\mu}(\mu=0, \ldots, \operatorname{dim}(X)-1)$ :

$$
\partial_{a}\left(\sqrt{-|h|} h^{a b} x_{, b}^{\nu} g_{\nu \mu}\right)-\frac{1}{2} \sqrt{-|h|} h^{a b} x_{, a}^{\rho} x_{, b}^{\nu} \frac{\partial g_{\rho \nu}}{\partial x^{\mu}}=0
$$


By $\Gamma_{\rho \nu}^{\mu}$ we denote the Christoffel symbols of $g$ and by $\square:=\eta^{a b} \partial_{a} \partial_{b}$ the flat wave operator. In conformal coordinates we have $\sqrt{-|h|} \cdot h^{a b}=\Lambda \cdot\left(\frac{1}{\Lambda} \eta^{a b}\right)=\eta^{a b}$, and (2.3) simplifies to

$$
\square x^{\mu}+\Gamma_{\rho \nu}^{\mu}(x) x_{, a}^{\rho} x_{, b}^{\nu} \eta^{a b}=0 . \quad \mu=0, \ldots, \operatorname{dim}(X)-1 .
$$

In slight generalization of notation we call a map $i:[0, a] \times S^{1} \rightarrow X$ a solution of (2.4), if any chart representation $x^{\mu}(\tau, \sigma)$ of $i$ obeys (2.4). The next lemma is standard in string theory, at least when $(X, g)$ is Minkowski space. For an elementary proof in the general case we refer to [4].

Lemma 2.1: Given a $C^{2}$-solution $i:[0, a] \times S^{1} \rightarrow X$ of the system (2.4) that obeys (2.1) at $\tau=0$, then (2.1) is satisfied for all $\tau \in[0, a], \sigma \in[0,2 \pi) \bmod 2 \pi$.

Suppose such a solution $i$ is given with the additional condition, that $\left.i\right|_{\{0\} \times S^{1}}$ is a spacelike imbedding, so we have $x^{\prime 2}(0, \sigma)>0$ for all $\sigma$. Then ${x^{\prime}}^{2}>0$ remains valid on $[0, \epsilon] \times S^{1}$, and for $\epsilon$ small enough, the parameters $(\tau, \sigma) \in[0, \epsilon] \times S^{1}$ are automatically conformal parameters on $\Sigma_{l o c}:=i\left([0, \epsilon] \times S^{1}\right)$, due to Lemma 2.1. Because (2.4) holds, $\Sigma_{l o c}$ is indeed a minimal surface.

Note that $\partial_{a} \sqrt{-|h|}$ and $h^{a b}$ in (2.3) are singular at points $p \in X$ where $|h|(p)=0$, but these singular terms completely drop out in the representation (2.4). This fact allows us to handle the singularities of (2.3) in a natural way: We simply consider the Cauchy evolution $i$ of initial configurations $\gamma$ by (2.4). The evolution may yield $|h|=0$ at certain points, so there are problems with (2.3), but nevertheless the map $i$ is well-defined. This extends the notion of a timelike minimal surface $\Sigma$ to an amount that is necessary from the viewpoint of time evolution, because the case $|h|=0$ already arises in simple examples, e.g. (4.7) below.

At points $(\tau, \sigma)$ with $x^{\prime 2}=-\dot{x}^{2} \neq 0$ the map $i$ is a local immersion in a neighbourhood of $(\tau, \sigma)$ but $i$ need not be a global immersion. If $x^{\prime 2}$ vanishes, the image $\Sigma=i(M)$ can have edges or cusps at those points. This behaviour is in sharp contrast to the smoothness properties of minimal surfaces, immersed in an Euclidean space. It is due to the fact that an area element im Minkowski space can have zero (two-dimensional) volume: Combining such two elements with an edge in common gives a small surface with zero volume; smoothing out this edge can only increase the volume. An example with a cusp (that approximates the cusp of a light cone) is given by (4.7) below, cf. also examples in [5].

\section{The geometric Cauchy problem}

Consider a spacelike $C^{2}$-submanifold $\gamma \cong S^{1}$ in $X$. It can be described as image under a $C^{2}$-imbedding $i_{0}$ of $S^{1}$ into $X$ with parameters $\sigma=\sigma \bmod 2 \pi$ on $S^{1}$. Along $\gamma$, we are given an orientable* $C^{1}$-distribution of timelike tangent planes, i.e. there exists a $C^{1}$-map $v$ from $S^{1}$ into $T X$, such that $v(\sigma) \in T_{i_{0}(\sigma)} X$ is a timelike vector and the tangent plane in $i_{0}(\sigma)$ is generated by $\frac{d}{d \sigma} i_{0}(\sigma)$ and $v(\sigma)$. The geometric Cauchy problem is now to find

\footnotetext{
* We need this condition to exclude surfaces like a Möbius strip.
} 
a minimal surface $\Sigma \supset \gamma$ whose tangent planes on $\gamma$ contain the vectors $v(\sigma)$. We call a solution $\Sigma$ locally unique if any other solution $\Sigma^{\prime}$ coincides with $\Sigma$ in a neighbourhood of $\gamma$ in $X$.

Theorem 3.1: Given a spacelike $C^{2}$-submanifold $\gamma \cong S^{1}$ in a Lorentz manifold $(X, g)$, and an orientable $C^{1}$-distribution of timelike tangent planes along $\gamma$; then there exists a $C^{2}$-solution $\Sigma$ of the geometric Cauchy problem; $\Sigma$ is locally unique.

Proof: There exists a $C^{2}$-imbedding $j: M_{\epsilon}=[0, \epsilon] \times S^{1} \rightarrow X$ with $j(0, \sigma)=i_{0}(\sigma)$, having the given distribution of tangent planes along $\gamma$. We equip $M_{\epsilon}$ with conformal $(\tau, \sigma)$ parameters as in Prop.1.2, and denote a chart representation of $j$ around $j(\tau, \sigma) \in X$ by $x^{\mu}(\tau, \sigma)$. The vectors $x^{\prime \mu}(0, \sigma), \dot{x}^{\mu}(0, \sigma)$ generate the given tangent plane at $i_{0}(\sigma)$ and in addition they satisfy the conformal gauge condition (2.1) at $\tau=0$. A unique $C^{2}$. solution of (2.4) with respect to these initial data exists (see [6]) in a neighbourhood of $\gamma$ on each chart representation $x^{\mu}$. These solutions glue together on overlapping charts and they define (because of Lemma 2.1) a minimal surface $\Sigma$ in a neighbourhood of $\gamma$. Hence existence holds.

For uniqueness, consider another solution $\Sigma^{\prime}$. We parametrize $\gamma \subset \Sigma^{\prime}$ by the same map $i_{0}$ as before. Applying the construction of Prop.1.2 for $\Sigma^{\prime}$ gives conformal $\left(\tau^{\prime}, \sigma^{\prime}\right)$ parameters on $\Sigma^{\prime}$ with $\sigma=\sigma^{\prime}$ at $\tau=\tau^{\prime}=0$. The tangent planes of $\Sigma$ and $\Sigma^{\prime}$ coincide along $\gamma$, implying $x_{, \tau^{\prime}}^{\mu}\left(0, \sigma^{\prime}\right)=x_{, \tau}^{\mu}(0, \sigma)$ for $\sigma=\sigma^{\prime}$. Therefore, both surfaces $\Sigma, \Sigma^{\prime}$ are determined by the same analytical Cauchy problem, hence they coincide locally.

Global existence for nonlinear hyperbolic equations is in general a much more difficult problem. The solution of (2.4) exists at least for small values $\tau \in(-\epsilon, \epsilon)$, denoted by $\Sigma_{l o c}$. When $\Sigma_{l o c}$ extends to all values $\tau \in \mathbb{R}$. we call the resulting surface a global solution $\Sigma$ of the geometric Cauchy problem. If global existence holds for one conformal parametrization of $\Sigma_{l o c}$, then it also holds for any other conformal parametrization which is induced by a diffeomorphism $\psi: S^{1} \rightarrow S^{1}$, as discussed after Prop.1.2; we skip the proof of that fact. For a string, the conservation law of Lemma 2.1 leads to global existence:

Theorem 3.2: Let the Lorentz manifold $(X, g)$ be defined by $X=\mathbb{R} \times N$ and $d s^{2}=$ $-d t^{2}+r_{i j}(x) d x^{i} d x^{j}$, where $(N, r)$ denotes a complete Riemannian manifold. Then the geometric Cauchy problem with initial data on $\gamma \check{C}\{0\} \times N$ has a global solution.

Proof: From Theorem 3.1 we get a local solution $\Sigma_{l o c}$ in conformal $(\tau, \sigma)$-parameters. We reparametrize the initial data to simplify the time component $\left(x^{0}=t\right)$ of $(2.4)$ as follows: The timelike vector $v^{\mu}(\sigma)=\dot{x}^{\mu}(0, \sigma)$ has a nonvanishing zero-component $\dot{t}(0, \sigma)=$ : $f(\sigma)$ for all $\sigma \in[0,2 \pi) \bmod 2 \pi$. Therefore,

$$
\tilde{\sigma}(\sigma):=\frac{1}{E} \int_{0}^{\sigma} f(s) d s
$$

defines another $2 \pi$-periodic parametrization of $\gamma$, with (energy) $E:=\frac{1}{2 \pi} \int_{0}^{2 \pi} f(s) d s$. The vectors $x_{, \tilde{\sigma}}^{\mu}(0, \tilde{\sigma})=\frac{E}{f(\sigma)} x_{, \sigma}^{\mu}(0, \sigma)$ and $\tilde{v}^{\mu}(\tilde{\sigma})=\frac{E}{f(\sigma)} v^{\mu}(\sigma)$ generate the same distribution of tangent planes along $\gamma$. At $\tau=0$ the equation $(2.1), \dot{x}^{2}+x^{\prime 2}=0$, transforms into

$$
-E^{2}+r_{i j} \tilde{v}^{i} \tilde{v}^{j}+r_{i j} x_{, \tilde{\sigma}}^{i} x_{, \tilde{\sigma}}^{j}=0
$$


We thus obtain a geometrically equivalent initial value problem with $\tilde{v}^{0}(0, \tilde{\sigma})=E$. The choice of $d s^{2}$ implies $\Gamma_{\mu \nu}^{0}=0$, so the zero component of (2.4) simplifies to $\square t=0$. The solution $\operatorname{reads} t(\tilde{\tau}, \tilde{\sigma})=E \tilde{\tau}$. Using Lemma 2.1 we find that (3.1) is conserved by the time evolution. In particular $r_{i j}(x) x_{, \tilde{\tau}}^{i} x_{, \tilde{\tau}}^{j} \leq E^{2}$ and $r_{i j}(x) x_{, \tilde{\sigma}}^{i} x_{, \tilde{\sigma}}^{j} \leq E^{2}$ hold. These pointwise estimates imply that the local solution $x^{i}(\tilde{\tau}, \tilde{\sigma})$ of $(2.4)$ extends to all values $\tilde{\tau} \in \mathbb{R}$.

Note that the solution exists for all values of the physical time parameter $t=E \cdot \tilde{\tau}$. We do not claim uniqueness in the global case (the uniqueness proof fails at points where $|h|(p)=0)$. A discussion of possible branching effects will be given elsewhere, cf. [7].

\section{A minimal surface in Schwarzschild spacetime}

Let $(X, g)$ be Schwarzschild spacetime, i.e. a manifold $X=\mathbb{R} \times(\mathbb{R}+\backslash\{2 M\}) \times S^{2}$ with a line element $d s^{2}=-\left(1-\frac{2 M}{r}\right) d t^{2}+\left(1-\frac{2 M}{r}\right)^{-1} d r^{2}+r^{2}\left(\sin ^{2} \theta d \varphi^{2}+d \theta^{2}\right)$, where $\varphi$ and $\theta$ are the sphere coordinates on $S^{2}$. Let $\gamma \subset{ }^{r} X$ be a circle, defined by $i_{0}(\sigma)=\left(0, R, \sigma, \frac{\pi}{2}\right)$, with $\sigma \in[0,2 \pi)$ and $R>2 M$ fixed. We choose $(\dot{t}, \dot{r}, \dot{\varphi}, \dot{\theta})(0, \sigma)=(E, 0,0,0)^{*}$ and the requirement $\dot{x}^{2}+x^{\prime 2}=0$, at $\tau=0$, yields $E=R / \sqrt{f(R)}$ with $f(r):=1-\frac{2 M}{r}$. The relation $\dot{x} x^{\prime}=0$ also holds so the conformal gauge (2.1) is satisfied for $\tau=0$.

In order to solve (2.4) for these initial data we make an ansatz: $t=t(\tau), r=r(\tau), \varphi=\sigma$ and $\theta=\frac{\pi}{2}$. We obtain

$$
\begin{gathered}
-\ddot{t}=\frac{2 M}{r(r-2 M)} \dot{t} \dot{r} \\
-\ddot{r}=\frac{1}{2} f^{\prime}(r) f(r) \dot{t}^{2}-\frac{1}{2} \frac{f^{\prime}(r)}{f(r)} \dot{r}^{2}+r f(r)
\end{gathered}
$$

from (2.4) as well as the equations $\square \varphi=0$ and $\square \theta=0$ which are satisfied by the ansatz. So we are left to solve (4.1) and (4.2). If our ansatz is correct, we have the conservation law $\dot{x}^{2}+x^{\prime 2}=0$ which is rewritten as

$$
f(r) \dot{t}^{2}=\frac{1}{f(r)} \dot{r}^{2}+r^{2}
$$

Replacing $f(r) \dot{t}^{2}$ in (4.2) by the right hand side of (4.3) yields a cancellation of all nonlinear terms! We find the equation $-\ddot{r}=r-M$ (a harmonic oscillator) which is solved by

$$
r(\tau)=(R-M) \cos \tau+M
$$

Equation (4.3) also gives $f(r) \dot{t}=\sqrt{\dot{r}^{2}+r(r-2 M)}=\sqrt{R(R-2 M)}$. The last equation is due to energy conservation of the harmonic oscillator. With $k:=\sqrt{R(R-2 M)}$ we obtain

$$
\dot{t}(\tau)=k \frac{r(\tau)}{r(\tau)-2 M}
$$

* These initial data describe a string at rest, lying at a "distance" $R>2 M$ from the singularity $r=0$. 
We have to check (4.1) for a solution of this equation, because the ansatz might fail. We differentiate (4.5), $\ddot{t}=-k \frac{2 M}{(r-2 M)^{2}} \dot{r}=-\frac{2 M}{r(r-2 M)} \dot{r} \cdot k \frac{r}{r-2 M}$ and replace the second factor by $\dot{t}$ to obtain $-\ddot{t}=\frac{2 M}{r(r-2 M)} \dot{t} \dot{r}$. Therefore, any solution of (4.5) satisfies (4.1). An elementary integration yields

$$
t(\tau)=\tau \cdot R \sqrt{1-\frac{2 M}{R}}+2 M \cdot \ln \left|\frac{\sqrt{1-\frac{2 M}{R}}+\tan \frac{\tau}{2}}{\sqrt{1-\frac{2 M}{R}}-\tan \frac{\tau}{2}}\right|
$$

Note that (4.4) and (4.6) satisfy the initial conditions so the problem is solved completely. The reader may compare this result with a particle falling without angular momentum into a black hole, e.g. in [8], p. 666. The singular term in (4.6) coincides with the singular term of the particle motion given there.

The Schwarzschild radius $r_{s}=2 M$ is reached by the string in the limit $\tau \rightarrow \tau_{s}=$ $\cos ^{-1}\left(\frac{M}{R-M}\right)$, and $t(\tau)$ tends to infinity for $\tau \rightarrow \tau_{s}$. At $\tau_{\max }=\cos ^{-1}\left(\frac{M}{M-R}\right)$ the string meets the singularity $r=0$. It is not hard to verify in Kruskal-Szekeres coordinates that (4.4) and (4.6), together with $\varphi=\sigma$ and $\theta=\frac{\pi}{2}$, define a smooth surface in Kruskal spacetime for all $\tau \in\left[0, \tau_{\max }\right)$. We observe that the domain of existence $\left[0, \tau_{\max }\right) \times S^{1}$ cannot be extended beyond $\tau_{\max }$.

Replacing $M$ by zero in (4.4) and (4.6) yields $t=R \tau, r=R \cos \tau, \varphi=\sigma$ and $\theta=\frac{\pi}{2}$. In Minkowski space $\left(\mathbb{R}^{3}, \operatorname{diag}(-1,1 ; 1)\right)$ (we suppress the constant z-component) this solution coincides with a well-known oscillating solution of period $\Delta \tau=\pi$ :

$$
(t, x, y)=(R \tau, R \cos \tau \sin \sigma, R \cos \tau \cos \sigma) \quad \forall \tau \in \mathbb{R}
$$

Note that the surface $\Sigma$ defined by this smooth solution has cusps at $\tau_{n}=\frac{\pi}{2}+n \pi$, and the "observable" oscillation period $\Delta t=R \pi$ tends to zero for $R \rightarrow 0$.

\section{Final comments}

1. We have seen that global existence holds for special types $(X, g)$ but not in general. Are there sufficient conditions on $(X, g)$ (e.g. boundedness of curvature etc.) that guarantee global existence? This problem is similar to the problem of geodesic completeness and both might be connected: One can argue heuristically that a small string moves approximately along a geodesic, performing rapid oscillations on its way, like in (4.7) (the smaller the string, the higher the frequency). Therefore, geodesic completeness of $(X, g)$ may be a necessary condition for global existence.

2. The Cauchy evolution of $\gamma$ respects the conformal gauge $i^{*} g=\Lambda \cdot \eta$, with $\Lambda=-\dot{x}^{2}$, and $\Lambda(p)=0$ occurs in several examples. In principle, $\Lambda$ might change sign, reversing the role of $\dot{x}$ as timelike vector and $x^{\prime}$ as spacelike vector. Then the curves $i(\tau, \cdot)$ ( $\tau$ fixed) will not be spacelike curves for all values of $\tau$, hence the interpretation of $\tau$ as a time-evolution parameter for a physical string gets lost. Note that this does not happen in the special case $X=\mathbb{R} \times N$ (cf. chapter 3 .). 
3. In chapter 1, we assumed $\Sigma=i(M)$ to be a timelike surface, from which we concluded $M \cong[0,1] \times S^{1}$. This conclusion fails if the induced metric $i^{*} g$ degenerates at certain points and indeed, there are minimal surfaces of higher topological type, describing splitting and merging effects of strings on a classical (i.e. non-quantized) level. A discussion of such surfaces in Minkowski space and their relation to the so-called Krichever-Novikov theory [9] is given in [7].

\section{Acknowledgement}

I thank Günter Schwarz for helpful discussions. This work was supported in part by the GradFöG Fund of Baden-Württemberg.

\section{References}

1. L. Brink and M. Henneaux, Principles of String Theory, Plenum Press, New York, (1988).

2. J. Douglas, Minimal surfaces of higher topological structure, Ann. of Math. 40, 205-298 (1939).

3. Ademollo M. et al., Theory of an Interacting String and Dual-Resonance Model, Il Nuovo Cimento 21A, 77-147 (1974).

4. Gu C.H., On the motion of a string in a curved space-time, in: Hu Ning (ed), Proc. 3rd. Grossmann meeting on General Relativity, Beijing, 139-142, (1983).

5. Turok N., Grand Unified Strings and Galaxy Formation, Nucl. Phys. B242, 520-541 (1984).

6. Courant R., Hilbert D., Methods of Mathematical Physics vol.2, Interscience, New York, (1962).

7. T. Deck, Classical string dynamics with non-trivial topology, Mannheimer Manuskripte 148/93, (1993).

8. Misner C.W., Thorne K.S., Wheeler J.A., Gravitation, Freeman and Company, San Francisco, (1970).

9. I.M. Krichever and S.P. Novikov, Virasoro Type Algebras, Riemann Surfaces and Strings in Minkowski Space, Funk. Anal. i Prilozhen 21 (4), (1987) 47-61. 